\title{
Clinical and microbiological characterization of KPC- producing Klebsiella pneumoniae infections in Brazil
}

\begin{abstract}
In 2008 isolates of KPC-producing Klebsiella pneumoniae (KPC-KPN) were detected for the first time at Hospital Heliópolis, São Paulo, Brazil. The aim of this study was to characterize the clinical and microbiological outcomes of infections caused by KPC-KPN. A historical cohort of patients from whom KPC-KPN strains were isolated was performed. Isolates were identified as resistant to ertapenem by automated broth microdilution system and screened as carbapenemase producers by the modified Hodge test. The beta-lactamase resistance gene blaKPC was detected by PCR. The genetic relatedness of isolates was determined by PFGE. The study provides early clinical experience in treating KPC-KPN infections in a Brazilian tertiary center.
\end{abstract}

Keyword: Klebsiella pneumonia; KPC; hospital infection; carbapenemase.

[Braz J Infect Dis 2011;15(1):69-73]@Elsevier Editora Ltda.

\section{INTRODUCTION}

Carbapenems such as imipenem and meropenem are recommended as first-line therapy for severe infections caused by Enterobacteriaceae producing extended spectrum $\beta$-lactamases (ESBLs). ${ }^{1}$ The increasing incidence of Klebsiella pneumoniae carbapenemase (KPC)-producing strains worldwide has posed a challenge for diagnosing and treating such infections since these isolates might be phenotypically detected as susceptible to carbapenems. ${ }^{2}$ In addition, therapeutic options for the treatment of these infections are basically restricted to tigecycline and polymyxins. However, the clinical use of these antimicrobials has been limited due to their pharmacokinetics properties and toxicity. Moreover, no new antibiotics against these multidrug-resistant (MDR) organisms are in advanced stages of clinical development (Table 1). ${ }^{3}$

KPC-producing Klebsiella pneumoniae (KPC-KPN) was first isolated in North Carolina in 1996. ${ }^{4}$ It remained infrequently isolated until 2001, when several outbreaks occurred in hospitals located in the New York region. ${ }^{5}$ KPCKPN has spread over time in many countries around the world, and have been frequently isolated from Israel, ${ }^{6}$ China $^{7}$ and Greece. ${ }^{8}$ In South America, the first detection of a KPC-2-producing Klebsiella pneumoniae isolates occurred in Colombia in 2006. Although the KPC-KPN isolates were detected in the cities of Recife and Rio de Janeiro, in 2006 and 2007, respectively, ${ }^{10,11}$ an early dissemination of KPC-KPN in the Brazilian territory was reported by Pavez et al. These authors showed that KPC-KPN was detected in the city of São Paulo, in 2005..$^{12}$ In 2008, we detected for the first time isolates of $K$. pneumoniae resistant to ertapenem in the Hospital Heliopolis, a teaching tertiary hospital located in the city of São Paulo, Brazil. These isolates were identified as resistant to ertapenem by automated broth microdilution system (MicroScan; Siemens) and screened as carbapenemase producers by the modified Hodge test as recommended by the Clinical Laboratory Standards Institute (CLSI, 2009). These isolates were referred to Laboratório Alerta, Division of Infectious Diseases, UNIFESP/EPM, where antimicrobial susceptibility testing, KPC production and genetic relatedness among KPC-KPN isolates were performed by CLSI agar dilution, PCR and PFGE, respectively.

We sought to study the clinical and microbiological outcomes of the KPC-KPN infections by performing a historical cohort of patients from whom KPC-KPN strains had been isolated. Medical records of colonized/infected KPC-KPN patients were reviewed. Success or failure of treatment was determined by clinical response. This study was approved by the institutional review board.
Authors

Elisa Maria Beirão ${ }^{1}$

Juvencio Jose Duailibe Furtado ${ }^{2}$

Raquel Girardello ${ }^{3}$ Heli Ferreira Filho ${ }^{4}$ Ana Cristina Gales ${ }^{5}$

${ }^{1} \mathrm{MD}, \mathrm{MSc}$, Infectious Diseases Physician of Hospital Heliópolis ${ }^{2}$ Chief of the Department of Infectious Diseases of Hospital Heliópolis ${ }^{3} \mathrm{PhD}$ Student in Infectious Diseases at, EPM/UNIFESP ${ }^{4}$ Chief of the Microbiology Laboratory of Hospital Heliópolis

${ }^{5} \mathrm{MD}, \mathrm{MSc}, \mathrm{PhD}$,

Coordinator of the Special Microbiology Laboratory of the Infectious Diseases Division, EPM/UNIFESP

Submitted on: 03/31/2010 Approved on: 06/18/2010

Correspondence to: Elisa Maria Beirão Departamento de Infectologia Hospital Heliopolis Rua Cônego Xavier,276 São Paulo, SP - CEP: 04231-030

Phone/Fax: 55-11 20636657 elisa.m.beirao@gmail.com

We declare no conflict of interest. 
Six KPC-KPN were isolated from six distinct patients between May 2008 and February 2009. Patient's age ranged from 54 to 89 years. Most patients $(66.6 \%)$ had been hospitalized for at least three weeks before KPC-KPN isolation. Beta-lactams (50\%) were the most common antimicrobial therapy prior to KPC-KPN isolation, with a single patient receiving carbapenem therapy.

The susceptibility profile of the KPC-KPN isolates is shown in Table 2. Of the six KPC-KPN cultured from inpa- tients, three (50\%) were originally reported as imipenem susceptible. Phenotypic detection of carbapenemase production was reported only three days later. All KPC-KPN exhibited resistance to beta-lactam/beta-lactamase inhibitor combination, carbapenems, and third-generation cefalosporins, except for a single isolate that showed susceptibility to ceftazidime and ceftriaxone. In contrast, all KPC-KPN isolates were susceptible to amikacin. No full resistance to tigecycline or polymyxin B was observed among the CRKP tested.

Table 1. Demographic characteristics and clinical outcomes of patients with KPC-KPN isolated

\begin{tabular}{|c|c|c|c|c|c|c|c|c|c|c|c|}
\hline $\begin{array}{l}\text { Patient } \\
\text { num- } \\
\text { ber }\end{array}$ & $\begin{array}{c}\text { PFGE } \\
\text { pat- } \\
\text { terns }\end{array}$ & $\begin{array}{c}\text { Sex/age } \\
\text { (years) }\end{array}$ & $\begin{array}{l}\text { Underlying } \\
\text { condition }\end{array}$ & $\begin{array}{l}\text { Comorbid } \\
\text { condition }\end{array}$ & $\begin{array}{l}\text { LOS } \\
\text { (days) }\end{array}$ & $\begin{array}{l}\text { Previous } \\
\text { antibiotics, } \\
\text { (days) }\end{array}$ & $\begin{array}{c}\text { Source } \\
\text { infection }\end{array}$ & $\begin{array}{l}\text { Antibiotic } \\
\text { treatment }\end{array}$ & $\begin{array}{l}\text { Leukocyte } \\
\text { urine/ } \\
\text { urinary } \\
\text { catheter } \\
\text { removal } \\
\text { (Yes/No) }\end{array}$ & $\begin{array}{c}\text { Clinical } \\
\text { signs } \\
\text { of sepsis }\end{array}$ & $\begin{array}{l}\text { Clinical } \\
\text { outcome }\end{array}$ \\
\hline 1 & A & $\mathrm{M} / 46$ & $\begin{array}{c}\text { Acute } \\
\text { abdominal } \\
\text { perforation }\end{array}$ & & 20 & $\begin{array}{c}\text { Ceftriaxone (14) } \\
\text { Metronidazole } \\
\text { (14) }\end{array}$ & Blood & Polymyxin B & - & Yes & $\begin{array}{l}\text { Death, } \\
\text { related to } \\
\text { infection }\end{array}$ \\
\hline 2 & B & $\mathrm{F} / 69$ & CNS tumor & Cancer & 23 & - & Urine & Ciprofloxacin & $\begin{array}{c}>10^{6} \\
\text { No }\end{array}$ & No & Cured \\
\hline 3 & A & $\mathrm{F} / 54$ & UTI & $\begin{array}{c}\text { CHF, } \\
\text { COPD, RF }\end{array}$ & 3 & - & Urine & Ceftriaxone & $\begin{array}{c}6 \times 10^{5} \\
\text { Yes }\end{array}$ & Yes & $\begin{array}{l}\text { Death, not } \\
\text { related to } \\
\text { infection }\end{array}$ \\
\hline 4 & $\mathrm{C}$ & $\mathrm{M} / 59$ & CNS cancer & $\begin{array}{l}\text { Cancer, } \\
\text { steroids }\end{array}$ & 78 & $\begin{array}{c}\text { Meropenem (49) } \\
\text { Vancomycin (64) } \\
\text { Cefepime (11) }\end{array}$ & $\begin{array}{c}\text { CNS } \\
\text { catheter }\end{array}$ & Meropenem & - & Yes & $\begin{array}{r}\text { Death not } \\
\text { related to } \\
\text { infection }\end{array}$ \\
\hline 5 & A & $\mathrm{M} / 89$ & $\begin{array}{l}\text { Infected } \\
\text { foot ulcer }\end{array}$ & $\begin{array}{c}\text { Chronic } \\
\text { arterial } \\
\text { insufficiency }\end{array}$ & 33 & $\begin{array}{c}\text { Ciprofloxacin } \\
\text { (21) }\end{array}$ & Urine & Cefepime & $\begin{array}{c}4 \times 10^{5} \\
\text { Yes }\end{array}$ & No & Cured \\
\hline 6 & A & $\mathrm{F} / 72$ & CNS tumor & $\begin{array}{l}\text { Cancer } \\
\text { steroids }\end{array}$ & 18 & Cefepime (12) & Urine & Cefepime & $\begin{array}{c}16 \times 10^{4} \\
\text { Yes }\end{array}$ & No & Cured \\
\hline
\end{tabular}

M, male; F, female; CHF, congestive heart failure; CNS, central nervous system; COPD, chronic obstructive pulmonary disease; LOS, length of stay prior to culture positive; RF, renal failure; UTI, urinary tract infection.

Table 2. MIC of selected antimicrobial agents for KPC-KPN strains

\begin{tabular}{lcccccc}
\hline \multicolumn{7}{c}{ Patient and $\mathbf{M I C}(\boldsymbol{\mu g} / \mathbf{m L})$} \\
\hline Antibiotic & 1 & 2 & 3 & 4 & 5 & 6 \\
\hline Amikacin & 8 & $<4$ & $<4$ & $<4$ & $<4$ & 8 \\
\hline Polymyxin B & $<0.5$ & $<0.5$ & $<0.5$ & 4 & $<0.5$ & 2 \\
\hline Ciprofloxacin & $>2$ & $<0.25$ & $>2$ & $>2$ & $>2$ & $>2$ \\
\hline Levofloxacin & $>4$ & 2 & $>4$ & $>4$ & $>4$ & $>4$ \\
\hline Aztreonam & $>16$ & $>16$ & $>16$ & $>16$ & $>16$ & $>16$ \\
\hline Cefoxitin & $>16$ & 8 & $>16$ & $>16$ & $>16$ & $>16$ \\
\hline Tigecycline & 1 & 0.5 & 4 & 2 & 2 & 2 \\
\hline Ceftriaxone & $>32$ & 8 & $>32$ & $>32$ & $>32$ & $>32$ \\
\hline Ceftazidime & $>16$ & 8 & $>16$ & $>16$ & $>16$ & $>16$ \\
\hline Cefepime & $>16$ & $>16$ & $>16$ & $>16$ & $>16$ & $>16$ \\
\hline Imipenem & $>8$ & $>8$ & $>8$ & $>8$ & $>8$ & $>8$ \\
\hline Meropenem & $>8$ & $>8$ & $>8$ & $>8$ & $>8$ & $>8$ \\
\hline Piperacillin/Tazobactam & $>64 / 4$ & $>64 / 4$ & $>64 / 4$ & $>64 / 4$ & $>64 / 4$ & $>64 / 4$
\end{tabular}


Among the six KPC-KPN isolates, three PFGE patterns were observed and denominated patterns A, B and C. Four isolates were clonally related (pattern A), while two isolates were not, with one of them isolated from a patient who had been hospitalized in a different institution.

\section{CASE REPORTS}

\section{Clinical case 1}

In April 2008, a 46 year-old man was submitted to an exploratory laparotomy due to perforated peptic ulcer and sent to the ICU for recovery. The patient had dehiscence of the surgical wound and was reoperated. After receiving ceftriaxone $2 \mathrm{~g}$ twice daily, and metronidazol $500 \mathrm{mg}$ three times a day for 14 days, the patient developed Klebsiella pneumoniae bloodstream infection associated with septic shock, and antibiotic was changed for piperacillin-tazobactam. This pathogen was resistant to ertapenem, but susceptible to imipenem by the MicroScan. This finding drew our attention to the possibility of a KPC-KPN. Three days after the isolation of KPC-KPN, the Hodge's test confirmed the production of a carbapenemase, and polymyxin B $15.000 \mathrm{UI} / \mathrm{kg} /$ day was initiated. No clinical response was observed and the patient deceased nine days later.

\section{Clinical case 2}

In May 2008, a 69 year-old woman was admitted for an elective surgical resection of a central nervous system (CNS) tumor. In the post-operatory, the patient was admitted on the neurosurgery ICU, and remained in this unit for 11 days. After this period, the patient was sent to the neurosurgery ward, and five days later, she presented an urosepsis. The patient was not receiving any antibiotics and intravenous ciprofloxacin $400 \mathrm{mg}$ twice daily was initiated. Urine culture confirmed the pure growth of K. pneumoniae ( $>10^{5}$ colony unit forms), susceptible to ciprofloxacin but resistant to ertapenem. The urethral catheter was removed and ciprofloxacin therapy was maintained for seven days. The patient showed a complete recovery and was discharged from the hospital 38 days later.

\section{Clinical case 3}

A 54 year-old woman was admitted to the ICU with signals and symptoms of septic shock due to a urinary tract infection. She was started on ventilatory support and noradrenaline and ceftriaxone $1 \mathrm{~g}$ twice daily were prescribed. The patient had a history of being hospitalized in another hospital 15 days before the current clinical manifestation. After three days, a K. pneumoniae ( $>10^{6}$ UFC) resistant to ertapenem, imipenem and meropenem was isolated from the urine culture. At that time, the patient was asymptomatic and not dependent on vasopressive drugs or mechanical ventilation. Since the patient had an excellent clinical response, ceftriaxone treatment was completed in the internal medicine ward. However, the patient died due to cardiac and respiratory failure after 27 days of hospitalization.

\section{Clinical case 4}

In June 2008, a 59 year-old man was admitted to hospital for a CNS tumor resection. In the 5th PO day, the patient had a surgical complication with a secondary bowel perforation by the ventriculo-peritoneal shunt. A fecal peritonitis was diagnosed and the patient was submitted to abdominal surgery. The ventriculo-peritoneal shunt was removed and an external ventricular shunt (EVS) was placed on. A K. pneumoniae secondary meningitis occurred three days after the EVS placement. This pathogen was susceptible to broad-spectrum cephalosporins and cefepime $2 \mathrm{~g}$ three times a day was empirically prescribed and kept until the 11th day of therapy, when an Acinetobacter baumannii was isolated from the spinal fluid during a routine culture. The EVS was replaced and cefepime therapy was stopped. Meropenem ( $2 \mathrm{~g}$ three times a day) was initiated and despite the isolation of $A$. baumannii, vancomycin $1 \mathrm{~g}$ twice daily was added to the antimicrobial regimen because the patient had undergone many surgical procedures during his hospitalization. After 49 days of vancomycin and meropenem therapy, a K. pneumoniae resistant to imipenem, meropenem and ertapenem was recovered from the CNS device. The neurosurgery team considered the $K$. pneumoniae as a contaminant because it was not recovered from his cerebrospinal fluid. The antimicrobial therapy was not modified, and the patient died five months later due to a respiratory infection.

\section{Clinical case 5}

In August 2008, an 89 year-old male with chronic arterial insufficiency was admitted to the vascular surgery ward for treatment of an infected right foot ulcer. The patient was treated with ciprofloxacin $400 \mathrm{mg}$ twice daily, and submitted to surgical treatment due to common iliac artery occlusion. In the 32nd day of hospitalization, the patient had signals and symptoms suggestive of urinary tract infection; ciprofloxacin therapy was replaced by cefepime $2 \mathrm{~g}$ twice daily. Three days later, the results of urine culture became available and showed the growth of a KPC-KPN $\left(>10^{6} \mathrm{CFU} / \mathrm{mL}\right)$. Since the patient had become asymptomatic, cefepime therapy was kept and the patient was discharged from the hospital after completing seven days of therapy.

\section{Clinical case 6}

A 72 year-old female patient with previous history of CNS tumor was taken unconscious to the emergency unit. Despite of the patient's option for not receiving surgical treatment, tumor resection was performed with the family consent. The patient was admitted to the ICU, where she received prolonged mechanical ventilation and urinary catheterization. She received antibiotic therapy with cefepime $2 \mathrm{~g}$ twice daily due to a tracheobronchitis. She was extubated four days later due to rapid clinical improvement. A urine culture was collected before urinary catheter removal in spite of no 
infection evidence. A KPC-KPN ( $\left.>10^{5} \mathrm{CFU}\right)$ was recovered from urine and considered just a colonizer since the patient had no clinical manifestations of urinary tract infection. The patient did not receive any antibiotics and was discharged one day later from our hospital.

\section{DISCUSSION}

We described microbiological features and clinical outcomes of six patients from whom KPC-KPN strains were firstly isolated in our institution. KPC-KPN represents a real challenge, since microbiological detection of this resistant phenotype might be tricky. In addition, a few therapeutic options are available for treatment of such infections, and are basically restricted to polymyxins and tigecycline. ${ }^{3}$

In our study, a urinary catheter was placed in all four patients from whom KPC-KPN strains were isolated from urine. All of these patients also showed elevated leukocyte count in urine. It has been reported that the risk of developing bacteriuria increased by $5 \%$ for each additional day of urinary catheterization and $50 \%$ of the patients had acquired bacteriuria by the tenth day of catheterization. ${ }^{13}$ The distinction between urinary colonization and infection is difficult because many times patients hospitalized in ICUs are sedated or unconscious and are not able to provide clinical information about his/her symptoms. Just one of the four patients colonized/infected by KPC-KPN strains was conscious and confirmed the symptoms suggestive of infection. This patient was successfully treated with ciprofloxacin guided by the antimicrobial susceptibility testing results. Two other patients were also successfully treated with broad-spectrum cephalosporins in spite of no reliable in vitro activity of these agents against KPC-KPN strains. ${ }^{14}$ We believe that these patients were infected by KPC-KPN strains because there was a resolution of clinical symptoms after introduction of the antimicrobial regimen. The successful clinical outcomes experienced by the patients treated with beta-lactam antibiotics have been previously reported ${ }^{15}$ but the degree to which the antibiotic treatment contributed to these outcomes is difficult to ascertain. Good clinical response to antibiotics with no in vitro activity might have been achieved by the increased antimicrobial concentration in the urine. In addition, most of the patients had the urine catheter removed, and this is of crucial importance for a successful outcome. ${ }^{14}$ Antimicrobial treatment was not modified because the Hodge test's final result was available only after the third day of beta-lactam therapy, when the patients have already had a favorable clinical outcome.

The patient reported in the clinical case 1 had a severe KPCKPN infection with a fatal outcome that could be attributed to an inadequate empiric therapy with piperacillin/tazobactam, a delay in the introduction of polymyxin as well as the low dose regimen of the later agent. Therapy was only changed three days after the isolation of Gram-negative bacilli in the blood culture.
Currently, the therapeutic options for highly resistant, carbapenemase-producing organisms are limited. Polymyxin B and tigecycline both seem to have reliable efficacy. ${ }^{3}$ Polymyxin is a compound developed decades ago and previously abandoned due to its toxicity. It has been resurrected because it is the only available therapeutic option for treatment of some pan-resistant Gram-negatives infections. ${ }^{16}$ Tigecycline is a novel extended-spectrum minocycline derivative, approved by the American Food and Drug Administration (FDA) for treatment of complicated skin, intra-abdominal infections and community-acquired pneumonia, but not for bloodstream and/or urinary infections. ${ }^{17}$ Although KPC-producing isolates show higher tigecycline MICs than those of wild type bacterial isolates, tigecycline possesses potent in vitro activity against these pathogens. ${ }^{18}$ Clinical data also supports efficacy of tigecycline for treating MDR Gram-negative infections in body sites where tigecycline does not reach therapeutic levels when habitual dosage regimens are prescribed. ${ }^{19}$ In these situations, the use of combined therapy and/or higher doses of tigecycline are critical for maximal therapeutic effectiveness. ${ }^{20}$ This would be specially useful in patients with infections due to pathogens with reduced susceptibility to polymyxin, such as those presented in the clinical cases 4 and 6 .

Although our study describes a small number of heterogeneous patients at a single institution, our findings should provide early clinical experience in treating KPC-KPN infections in Brazil. Our results also underscore the importance of implementation of strict infection control measures to limit their dissemination, since four isolates were clonally related suggesting a patient-to-patient transmission in our institution.

\section{REFERENCES}

1. Paterson D L and Bonomo RA. Extended-Spectrum $\beta$-Lactamases: a Clinical Update.Clinical Microbiology Reviews 2005; 18(4):657-686.

2. Nordmann P, Cuzon G, Naas T. The real threat of Klebsiella pneumoniae carbapenemase producing bacteria. Lancet Infect Dis. 2009; 9:228-36.

3. Arias CA. and Murray BM. Antibiotic-Resistant Bugs in the 21st Century - A Clinical Super-Challenge. N Engl J Med 2009; 360:439-443.

4. Yigit H, Queenan AM, Anderson GJ et al. Novel carbapenemhydrolyzing beta-lactamase, KPC-1, from a carbapenemresistant strain of Klebsiella pneumoniae. Antimicrob Agents Chemother. 2001; 45:1151-1161.

5. Bratu S, Brooks S, Burney S et al. Detection and spread of Escherichia coli possessing the plasmid-borne carbapenemase KPC-2 in Brooklyn, New York. Clin Infect Dis. 2007; 44:972-975.

6. Leavitt A, Navon-Venezia S, Chmelnitsky I et al. Emergence of KPC-2 and KPC-3 in carbapenem-resistant Klebsiella pneumoniae strains in an Israeli hospital. Antimicrob. Agents Chemother. 2007; 51:3026-29. 
7. Wei ZQ, Du XX, Yu YS et al. Plasmid-mediated KPC-2 in a Klebsiella pneumoniae isolate from China. Antimicrob Agents Chemother. 2007; 51:763-65.

8. Cuzon G, Naas T, Demachy MC et al. Plasmid-mediated carbapenem-hydrolyzing $\beta$-lactamase KPC in Klebsiella pneumoniae isolate from Greece. Antimicrob Agents Chemother. 2008; 52:796-97.

9. Villegas MV, Lolans K, Correa A et al. First detection of the plasmid-mediated class A carbapenemase KPC-2 in clinical isolates of Klebsiella pneumoniae from South America. Antimicrob Agents Chemother. 2006; 50:2880-82.

10. Monteiro J, Santos AF, Asensi MD et al. First report of KPC2-producing-Klebsiella pneumoniae in Brazil. Antimicrob Agents Chemother. 2008; 53:333-34.

11. Peirano G, Seki LM, Val Passos VL et al. Carbapenem hydrolysing $\beta$-lactamase KPC-2 in Klebsiella pneumoniae isolated in Rio de Janeiro, Brazil. J Antimicrob Chemother. 2009; 63:265-68.

12. Pavez M, Neves P, Dropa M et al. Emergence of carbapenemresistant Escherichia coli producing CMY-2-type AmpC betalactamase in Brazil. J Med Microbiol. 2008; 57(Pt 12):1590-2.

13. Garibaldi RA, Burke JP, Dickman ML and Smith CB (1974). Factors predisposing to bacteriuria during indwelling urethral catheterisation. The New England Journal of Medicine 1974; 291:213-219.

14. Stephen G Jenkins; David P. Calfee, Outcomes of Carbapenem-Resistant Klebsiella pneumoniae Infection and the Impact of Antimicrobial and Adjunctive Therapies. Infect Control Hosp Epidemiol. 2008; 29:1099-1106.
15. Weisenberga SA, Morganc DJ, Espinal-Witterb R, Laroneb D.H. Clinical outcomes of patients with Klebsiella pneumoniae carbapenemase-producing K. pneumoniae after treatment with imipenem or meropenem. Diagnostic Microbiology and Infectious Disease 2009; 64:233-23

16. Landman D, Georgescu C, Martin DA, Quale J. Polymyxins Revisited. Clinical Microbiology Reviews 2008; 21(3):449465.

17. Hawkey P, Finch R. Tigecycline: in-vitro performance as a predictor of clinical efficacy. Clin Microbiol Infect. 2007; 13:354-62.

18. Castanheira M, Sader HS, Deshpande LM, Fritsche TR, Jones RN. Antimicrobial activities of tigecycline and other broad-spectrum antimicrobials tested against serine carbapenemase- and metallo-beta-lactamase-producing enterobacteriaceae: Report from the SENTRY antimicrobial surveillance program. Antimicrobial Agents and Chemotherapy 2008; 52(2):570-573.

19. Anthony KB, Fishman NO, Linkin DR, Gasink LB, Edelstein $\mathrm{PH}$, Lautenbach E. Clinical and Microbiological Outcomes of Serious Infections with Multidrug- Resistant Gram-Negative Organisms Treated with Tigecycline. Clinical Infectious Diseases 2008; 46:567-70.

20. Nicasio AM, Crandon JL, Nicolau DP. In vivo Pharmacodynamic Profile of Tigecycline against Phenotypically Diverse Escherichia coli and Klebsiella pneumoniae Isolates. Antimicrobial Agents and Chemotherapy 2009; 53:27562761. 